\title{
Application Range Analysis and Implementation of the Logic-Processed CPS-PWM Scheme Based MMC Capacitor Voltage Balancing Strategy
}

\author{
Kun Wang, Leyuan Zhou, Yan Deng, Yi Lu, Chaoliang Wang, and Feng Xu
}

\begin{abstract}
Fundamental frequency sorting algorithm (FFSA) is outstanding for the MMC capacitor voltage balance due to the reduced computational burden and elimination of arm current detection. However, with the traditional carrier-phase-shifted pulse width modulation (CPS-PWM) scheme, FFSA will be ineffective when the carrier frequency is higher than $250 \mathrm{~Hz}$, where the line frequency is $50 \mathrm{~Hz}$. Thus, previous works proposed a logicprocessed CPS-PWM scheme to overcome this disadvantage. This paper furtherly introduces the application ranges and implementation of this logic-processed CPS-PWM scheme based capacitor voltage balancing method in detail. With a detailed mathematical analysis, the factors that influence the balancing strategy's convergence speed are obtained, i.e., modulation index and power factor angle. It is found that in the applications where the modulation index is usually higher than 0.75 , the influence of the modulation index is negligible. However, when the power factor angle is closed to $\pm \pi / 2$, the convergence speed is almost zero. Therefore, by comparing with the traditional CPS-PWM scheme based FFSA balancing method, the application ranges of power factor angle are revealed to guarantee a high convergence speed. Meanwhile, the balancing strategy's application scope is specified. Moreover, a three-tier control architecture is demonstrated, where the logic process of driving signals and capacitor voltage sorting process are both executed in the middle-tier FPGA controller. This centralized scheme guarantees the synchronization of switching actions. And the logic process is easy and cost-effective in FPGA. Simulation and experimental results are presented to validate the theoretical analysis.
\end{abstract}

Index Terms-Balancing strategy, convergence speed, FFSA, logic-processed CPS-PWM, modular multilevel converter.

\section{INTRODUCTION}

$\mathrm{M}^{\mathrm{s}}$ ODULAR multilevel converter (MMC) has attracted great attention due to its modularity, high efficiency and scalability [1]-[3]. With these advantages, MMC is promising

Manuscript received January 22, 2019. This work is supported by National Key R\&D Program of China (2017YFB0903100), Science and Technology Projects of State Grid Corporation of China (521104170043). This paper was presented in part at the 2018 IEEE International Power Electronics and Application Conference and Exposition (PEAC), Shenzhen, China, November 2018.

K. Wang, L. Zhou, and Y. Deng are with the College of Electrical Engineering, Zhejiang University, Hangzhou 310027, China (e-mail: wkun@ zju.edu.cn; zlyuan@zju.edu.cn; dengyan@zju.edu.cn).

Y. Lu, C. Wang, and F. Xu are with the State Grid Zhejiang Electric Power Research Institute, Hangzhou 310014, China (e-mail: lu_yi51@zj.sgcc.com.cn; chaoliangwang@126.com; xuf_1988@163.com).

Digital Object Identifier 10.24295/CPSSTPEA.2019.00001 in medium- and high-voltage applications. Researches on modulation schemes, voltage balancing strategies and circulating current suppression strategies have been widely carried out [4] [7]. Capacitor voltage balancing of sub-modules (SMs) is vital for the stable operation and performance of MMC.

Different kinds of voltage balancing methods have been presented in the literature. Among them, the open-loop balancing method requires no measurement of the capacitor voltages, which simplifies the design of the control system and reduces the cost [8]. However, its dynamic performance is poor. Sorting and selecting method is one of the most commonly used methods, which sorts the capacitor voltages at first and then inserts proper SMs according to the sorting result and the arm current direction [9]. Although this method is easy to implement, the high frequency sorting process leads to heavy computational burden and excessive switching actions. A reduced switchingfrequency voltage balancing strategy was proposed in [10] to reduce the switching actions of SMs. But the sorting frequency of this method is still high. Fundamental frequency sorting algorithm (FFSA) based balancing strategy is proposed in [11], which avoids the excessive switching actions and effectively reduces the sorting frequency to fundamental frequency. So, a large amount of computing resources is saved. Further studies on FFSA have been carried out in [12]-[14] under different modulation schemes, such as carrier-phase-shifted pulse width modulation (CPS-PWM), nearest level (NL) modulation and phase disposition PWM (PD-PWM). And it is found in [14] that, with CPS-PWM scheme, FFSA based balancing strategy will no longer feasible when the carrier frequency is higher than $250 \mathrm{~Hz}$, where the line frequency is $50 \mathrm{~Hz}$. This is caused by the little difference in the charging ability among different driving signals. Therefore, an improved balancing strategy is proposed in [14]-[15] based on the logic-processed CPS-PWM scheme. In the improved balancing strategy, driving signals with proper phase angle difference are processed by logic $A N D / O R$ process to synthesize new driving signals with obviously charging ability difference. Subsequently, the FFSA can balance the capacitor voltage under high-frequency CPS-PWM. However, the convergence speed and application ranges of the proposed balancing strategy is not analyzed in detail.

In this paper, the convergence speed of logic-processed CPS-PWM scheme based MMC capacitor voltage balancing strategy is analyzed in detail. It is found that the convergence 


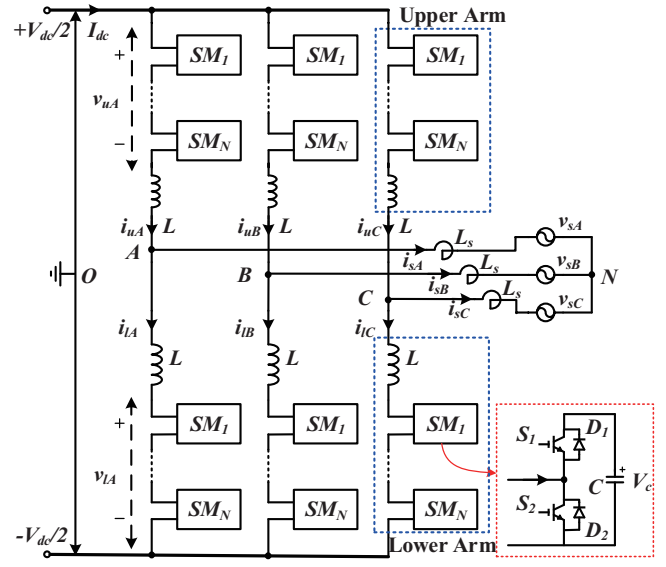

Fig. 1. Structure of a typical three-phase grid-connected MMC.

speed is influenced by the modulation index and power factor angle. While, as revealed in this paper, in the applications where the modulation index is usually higher than 0.75 , the influence of modulation index is negligible. However, when the power factor angle is closed to $\pm \pi / 2$, the balancing strategy becomes infeasible because the convergence speed is too slow to balance the capacitor voltage. By comparing with the traditional CPSPWM scheme based FFSA balancing method, the application ranges of power factor angle are revealed. Consequently, the proposed balancing method's application scope is specified. It is especially suitable for the high voltage direct current (HVDC) transmission, but not suitable for applications such as static synchronous compensator (STATCOM), which mainly generate or absorb reactive power. Moreover, a three-tier control architecture is also presented, where the logic-processed CPS-PWM is centralized in the middle-tier FPGA controllers for each phase. Thus, the synchronization of switching actions is guaranteed, and the logic process can be accomplished easily and cost-efficiently.

This paper is organized as follows. The principles of logicprocessed CPS-PWM scheme based capacitor voltage balancing strategy are presented in Section II. In Section III, the convergence speed is analyzed in detail, and the application ranges of power factor angle are revealed subsequently. The three-tier control architecture is demonstrated in Section IV. Simulation and experimental results verify the theoretical analysis in Section V. Finally, Section VI draws the conclusion.

\section{LOGIC-PROCESSED CPS-PWM SCHEME BASED Voltage Balancing Strategy With FFSA}

\section{A. Structure of $M M C$}

A typical structure of three-phase grid-connected MMC is illustrated in Fig. 1, where each phase leg is comprised of two arms. In each arm, $N$ SMs and one inductor are connected in series. The SM consists of two IGBT switches and one capacitor. When $S_{1}$ is on, the SM is inserted. And it is bypassed when $S_{2}$ is on. Thus, the output voltage of SM varies between $V_{\mathrm{c}}$ and zero, where $V_{\mathrm{c}}$ is the capacitor voltage. The output voltage

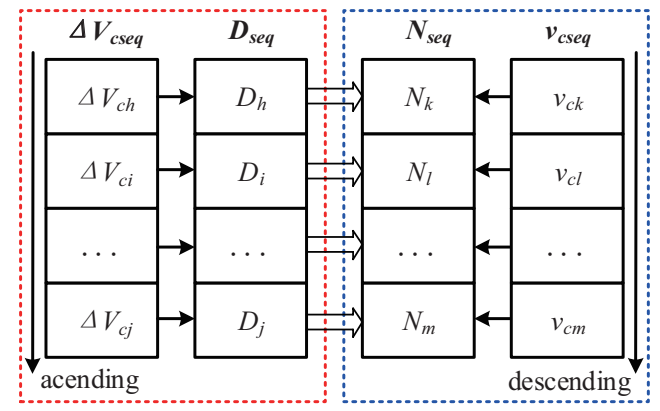

Fig. 2. Principle of fundamental frequency sorting algorithm.

can be synthesized by properly inserting a certain number of SMs in upper and lower arms.

\section{B. Traditional CPS-PWM Scheme Based Balancing Strategy With FFSA}

The basic principle of FFSA is firstly introduced in [11]. In this algorithm, the capacitor voltage variations of SMs in the previous fundamental period are calculated and sorted in ascending order at the fundamental frequency. In Fig. 2, $\Delta V_{c h}$, $\Delta V_{c i}, \Delta V_{c j}$ represent the voltage variations of $\mathrm{SM}_{h}, \mathrm{SM}_{i}$ and $\mathrm{SM}_{j}$, and $\Delta V_{c s e q}$ stores the sorting result. The voltage variations sorting result reflects the charging ability of each SM's driving signal assigned to them in the previous fundamental period. Subsequently, the driving signals sequence, namely $D_{\text {seq }}$, is obtained in ascending order regarding to their charging ability. Meanwhile, the capacitor voltages are sorted in descending order, obtaining the voltage sequence labeled as $v_{c s e q}$. Symbols $v_{c k}, v_{c l}$, and $v_{c m}$ represent the capacitor voltage of $\mathrm{SM}_{k}, \mathrm{SM}_{l}$ and $\mathrm{SM}_{m}$, respectively. And $N_{\text {seq }}$ stores the corresponding number of the SMs. So far, the relationship between driving signals and SMs can be rebuilt with the balancing strategy going like this: in the following fundamental period, driving signal which has the lowest charging ability will be assigned to SM with the highest capacitor voltage, e.g., $D_{h}$ is assigned to $\mathrm{SM}-\mathrm{N}_{k}$, while driving signal which has the highest charging ability will be assigned to $\mathrm{SM}$ with the lowest capacitor voltage, e.g., $D_{j}$ is assigned to SM$N_{m}$. Thus, the capacitor voltage can be well balanced.

However, in this scenario, the charging ability of driving signals is related to the CPS-PWM carrier frequency. As illustrated in Fig. 3, $N$ carriers are compared with the modulation signal $v_{\text {aref }}$ to generate driving signals for the lower arm of phase $A$. Taking $S_{x}$ as an example, which is generated by the $x^{\text {th }}$ carrier, the Fourier expression of $S_{x}$ can be derived as:

$$
\begin{aligned}
S_{l x C P S}= & \frac{1}{2}+\frac{m_{v}}{2} \cos \left(\omega t+\theta_{v}\right) \\
& +\sum_{m=1}^{\infty} \sum_{n=-\infty}^{\infty} A_{m n} \cos \left[m\left(\omega_{c} t+\theta_{c x}\right)+n\left(\omega t+\theta_{v}\right)\right]
\end{aligned}
$$

where $m_{v}$ is the modulation index, $\omega$ is the angular frequency of modulation signal, $\omega_{c}$ is the angular frequency of carrier, $\theta_{v}$ is the phase angle of the modulation signal, $\theta_{c x}$ is the initial phase angle of $x^{\text {th }}$ carrier and $A_{m n}$ is the amplitude of harmonic 


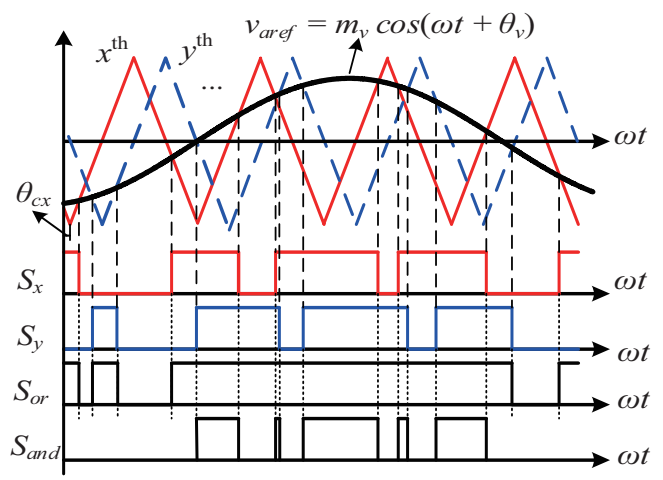

Fig. 3. Illustration of CPS-PWM and logic processing of driving signals.

component, in which $m$ is the carrier harmonic index and $n$ is the carrier sideband harmonic index $(m=1,2,3, \ldots$ and $n=0, \pm 1, \pm 2, \ldots)$. So the voltage variation caused by the $x^{\text {th }}$ carrier during one fundamental period can be calculated as:

$$
\Delta V_{c l x C P S}=\frac{1}{C} \int_{0}^{T} S_{l x C P S} \times i_{l A} d t=\Delta V_{1}+\Delta V_{2}+\Delta V_{3}
$$

where $i_{l A}$ is the lower arm current of phase $A$ and $\Delta V_{1}, \Delta V_{2}$ and $\Delta V_{3}$ represent the voltage variations caused by carrier harmonics and their sidebands that overlap the DC component, the fundamental component and the secondary harmonic, respectively. As presented in [14], if the line frequency is $50 \mathrm{~Hz}$, when the carrier frequency is higher than $250 \mathrm{~Hz}$, the charging abilities of each driving signal are all near to zero, which leads to the failure of balancing strategy based on traditional CPSPWM scheme with FFSA.

\section{Logic-Processed CPS-PWM Scheme Based Balancing Strategy With FFSA}

In order to synthesize driving signals with obviously different charging ability when the carrier frequency is higher than $250 \mathrm{~Hz}$, the logic-processed CPS-PWM scheme is proposed. As depicted in Fig. 3, carrier $x$ and carrier $y$ are selected in pairs as an example. Driving signal $S_{o r}$ is the logic $O R$ process result of $S_{x}$ and $S_{y}$, and $S_{\text {and }}$ is the result of logic $A N D$ process of $S_{x}$ and $S_{y}$. As proved in the next section, the logic-processed driving signals, $S_{\text {or }}$ and $S_{\text {and }}$ possess obviously different charging ability with each other. So the logic-processed CPS-PWM scheme based balancing strategy with FFSA can be executed as follows.

Firstly, the sorting process of capacitor voltages is operated at fundamental frequency with the corresponding SM's number sequence stored in $N_{\text {seq }}$, as illustrated in Fig. 4. The voltage differences of each SM-pair can be obtained simultaneously, where the SMs are combined in pairs by the following rules: $\mathrm{SM}$ with the highest capacitor voltage is combined with the SM with lowest capacitor voltage, and the second highest SM is combined with the second lowest SM, and so on. In this paper, $2 \%$ of the rated capacitor voltage is set as the threshold to determine whether the SMs are in balance state or not. If the voltage difference overreaches this threshold, it means that the corresponding SM-pair is in unbalance state. As shown in

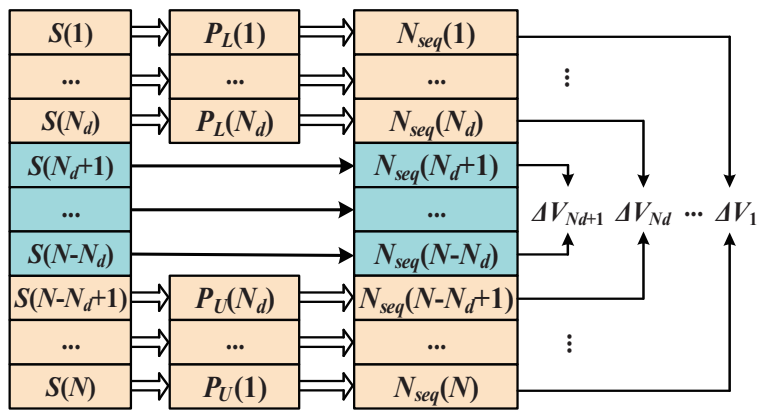

Fig. 4. Logic process and assignment of the driving signals.

Fig. 4, assume there are $N_{d}$ pairs of SMs in unbalance state, i.e., voltage differences of the highest $N_{d}$ SMs and the lowest $N_{d}$ SMs $\left(\Delta V_{1} \sim \Delta V_{N d}\right)$ exceed the threshold. Accordingly, $N_{d}$ pairs of driving signals with proper phase angle difference are selected in $S(i)$ and $\mathrm{S}(\mathrm{N}-i+1)$, where $i=1,2, \ldots, N_{d}$. And the rest driving signals are stored in $S\left(N_{d}+1\right)$ to $S\left(N-N_{d}+1\right)$, which are directly assigned to the SMs in balance state. $P_{L}(i)$ and $P_{U}(i)$ represent the logic process of driving signal pairs as defined in (3) and (4), where $\min \{\}$ means selection of the driving signal with lower charging ability, and $\max \{\}$ means selection of the driving signal with higher charging ability. And then, $P_{L}(i)$ is assigned to SM- $N_{\text {seq }}(i)$, while $P_{U}(i)$ is assigned to $\mathrm{SM}-N_{\text {seq }}(\mathrm{N}-i+1)$. Consequently, the capacitor voltages can converge back to the balance state.

$$
\begin{aligned}
& P_{L}(i)=\min \left\{\frac{S(i) \| S(N-i+1)}{S_{\text {or }}}, \frac{S(i) \& \& S(N-i+1)}{S_{\text {and }}}\right\} \\
& P_{U}(i)=\max \left\{\frac{S(i) \| S(N-i+1)}{S_{o r}}, \frac{S(i) \& \& S(N-i+1)}{S_{\text {and }}}\right\}
\end{aligned}
$$

\section{Convergence Speed and Application Range ANALYSIS}

It is clear that the charging ability difference between driving signals is the key to balance the capacitor voltages. The voltage balancing strategy may be deactivated when the charging ability difference is not so obvious. Thus, the convergence speed is analyzed in this section and the application range is revealed.

\section{A. Convergence Speed Analysis}

Similar to the traditional CPS-PWM scheme, the Fourier expression of $S_{o r}$ and $S_{\text {and }}$ can be obtained and the voltage variations caused by them in one fundamental period can be derived as follows:

$$
\begin{aligned}
\Delta V_{o r} \approx & -\frac{I_{s m}}{\omega C} \frac{m_{v}}{2} \cos \theta\left(m_{v} \sin \varphi_{x y}-\varphi_{x y}-\frac{\theta_{x y}}{2}+\frac{\theta_{x y} \varphi_{x y}}{\pi}\right) \\
& -\frac{I_{s m}}{\omega C} \frac{\varepsilon_{c 2 \omega}}{2} \cos \left(2 \theta_{v}-\theta_{c 2 \omega}\right) \\
& \times\left(m_{v} \sin \varphi_{x y}+\frac{\theta_{x y}-\pi}{\pi} \sin 2 \varphi_{x y}+\frac{m_{v}}{3} \sin 3 \varphi_{x y}\right)
\end{aligned}
$$




$$
\begin{aligned}
\Delta V_{a n d} \approx & \frac{I_{s m}}{\omega C} \frac{m_{v}}{2} \cos \theta\left(m_{v} \sin \varphi_{x y}-\varphi_{x y}-\frac{\theta_{x y}}{2}+\frac{\theta_{x y} \varphi_{x y}}{\pi}\right) \\
& +\frac{I_{s m}}{\omega C} \frac{\varepsilon_{c 2 \omega}}{2} \cos \left(2 \theta_{v}-\theta_{c 2 \omega}\right) \\
& \times\left(m_{v} \sin \varphi_{x y}+\frac{\theta_{x y}-\pi}{\pi} \sin 2 \varphi_{x y}+\frac{m_{v}}{3} \sin 3 \varphi_{x y}\right)
\end{aligned}
$$

where $I_{s m}$ is the amplitude of output phase current, $\theta$ is the power factor angle, $\theta_{x y}$ is the phase angle difference of carrier $x$ and $y$, and $\varphi_{x y}$ is calculated as follows:

$$
\varphi_{x y}=\left\{\begin{array}{lc}
0, & 0 \leqslant \theta_{x y} \leqslant\left(1-m_{v}\right) \pi \\
\arccos \frac{1-\theta_{x y} / \pi}{m_{v}}, & \left(1-m_{v}\right) \pi<\theta_{x y}<\left(1+m_{v}\right) \pi \\
\pi, & \left(1+m_{v}\right) \pi \leqslant \theta_{x y}<2 \pi
\end{array}\right.
$$

It should be noticed that the voltage variations caused by the carrier sideband harmonic are neglected in (6) since they are very small when the carrier frequency is high. Moreover, because the driving signals are logic processed in pairs and assigned to the SM-pairs simultaneously, the convergence speed of capacitor voltage can be evaluated by the difference of variations caused by $S_{o r}$ and $S_{\text {and }}$ as shown in (8).

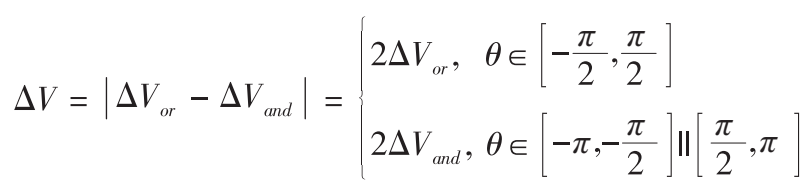

Furtherly, since the second part of (5) and (6) is also small enough to be neglected, only the first part of the voltage variations is considered for simplification. Thus, $\Delta V$ can be simplified as follows:

$$
\begin{aligned}
\Delta V & =2\left|\Delta V_{o r_{-} 1}\right|=2\left|\Delta V_{\text {and } \_1}\right| \\
& =\frac{I_{s m}|\cos \theta|}{\omega C} m_{v}\left(\varphi_{x y}+\frac{\theta_{x y}}{2}-m_{v} \sin \varphi_{x y}-\frac{\theta_{x y} \varphi_{x y}}{\pi}\right)
\end{aligned}
$$

It can be observed that the convergence speed is determined by active current component $I_{s m}|\cos \theta|$, modulation index $m_{v}$ and carrier phase angle difference $\theta_{x y}$. By the partial differential operation of $\theta_{x y}$ and $\varphi_{x y}$, the following equation can be obtained as:

$$
\left\{\begin{array}{l}
\frac{\partial \Delta V}{\partial \theta_{x y}}=\frac{I_{s m}|\cos \theta|}{\omega C} m_{v}\left(\frac{1}{2}-\frac{\varphi_{x y}}{\pi}\right) \\
\frac{\partial \Delta V}{\partial \theta_{x y}}=\frac{I_{s m}|\cos \theta|}{\omega C} m_{v}\left(1-m_{v} \cos \varphi_{x y}-\frac{\theta_{x y}}{\pi}\right)
\end{array}\right.
$$

Assuming (10) to be zero, it can be derived that $\Delta V$ achieves its maximum value when $\theta_{x y}=\pi$ and $\varphi_{x y}=\pi / 2$. Consequently, the maximum convergence speed $\Delta V_{\max }$ can be calculated as:

$$
\Delta V_{\max }=\frac{I_{s m}|\cos \theta|}{\omega C} m_{v}\left(\frac{\pi}{2}-m_{v}\right)
$$

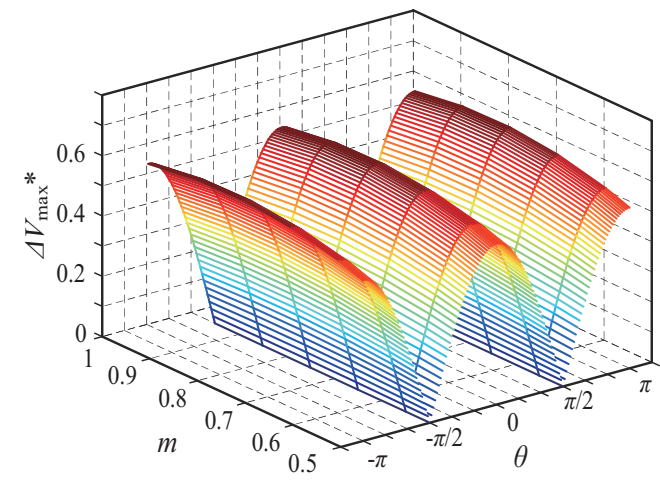

(a)

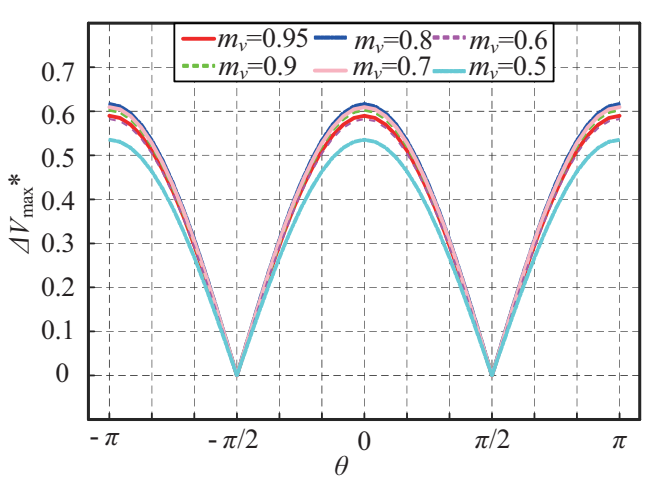

(b)

Fig. 5. Influence of power factor angle $\theta$ and modulation index $m_{v}$ on the normalized convergence speed $\Delta V_{\max }{ }^{*}$.

Normalized by $I_{s m} / \omega C$, the relationship of $\Delta V_{\max }{ }^{*}, m_{v}$ and $\theta$ is illustrated in Fig. 5. It can be observed that the balancing strategy has the highest convergence speed when $\theta$ equals to 0 or $\pm \pi$. While it reaches the lowest speed when $\theta$ equals to $\pm \pi / 2$. And it can be seen from Fig. 5(b) that when $m_{v}$ is higher than $0.75, m_{v}$ has little influence on the convergence speed. Thus, only the application range of power factor angle $\theta$ needs to be analyzed in the next subsection.

\section{B. Application Range Analysis}

It is presented in [14] that the balancing strategy's convergence speed will be degraded when there is reactive power transferred. However, the acceptable application range of power factor angle is not clearly specified. It is reasonable to take the convergence speed of traditional CPS-PWM based balancing strategy as the reference to analyze the application ranges of the proposed strategy. As demonstrated above, the traditional CPS-PWM based balancing strategy's convergence speed slows down with the increase of carrier frequency and eventually becomes infeasible when the carrier frequency is higher than $250 \mathrm{~Hz}$. But it can still offer considerable speed when the carrier frequency is $150 \mathrm{~Hz}$ [14]. Therefore, the convergence speed of traditional CPS-PWM based balancing strategy with $150 \mathrm{~Hz}$ carrier frequency is taken as the lowest acceptance of convergence speed for the proposed balancing strategy.

Based on the expansion of (2), the maximum and minimum 


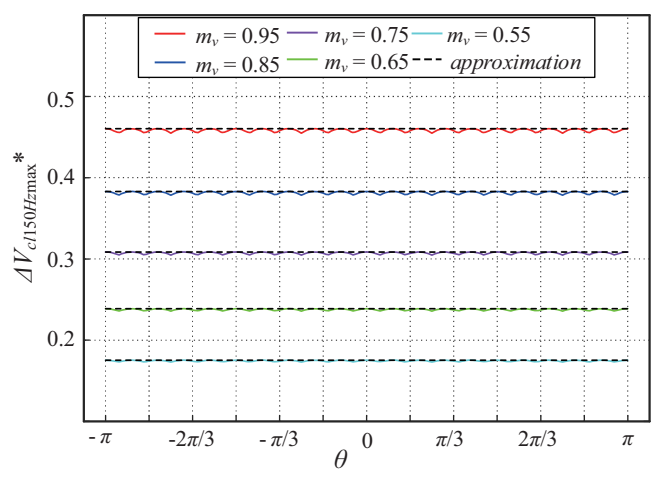

Fig. 6. Convergence speed $V_{c l 150 H z \max }{ }^{*}$ versus the power factor angle $\theta$ and the modulation index $m_{v}$

capacitor voltage variations generated by the driving signals with $150 \mathrm{~Hz}$ carrier frequency are calculated as (12).

$$
\left\{\begin{array}{l}
\Delta V_{c l \times 150 H z \max } \approx-\frac{I_{s m}}{\omega C} \frac{\pi}{2} \times A_{1(-2)} \cos \left(\theta_{c x 1}-3 \theta_{v}+\theta\right) \\
\Delta V_{c l \times 150 H z \min } \approx-\frac{I_{s m}}{\omega C} \frac{\pi}{2} \times A_{1(-2)} \cos \left(\theta_{c x 2}-3 \theta_{v}+\theta\right)
\end{array}\right.
$$

where $\theta_{c x 1}$ is the phase angle of carrier $x_{1}, x_{1}=\bmod$ (round $\left.\left(1+N\left(\theta_{c 1}-3 \theta_{v}+\theta\right) / 2 \pi\right), N\right), \theta_{c x 2}$ is the phase angle of carrier $x_{2}, x_{2}=\bmod \left(\operatorname{round}\left(1+N\left(\theta_{c 1}-3 \theta_{v}+\theta-\pi\right) / 2 \pi\right), N\right)$. And $A_{1(-2)}$ is the harmonic amplitude, which can be calculated as:

$$
A_{1(-2)}=\frac{1}{\pi^{2}} \int_{-\pi}^{\pi} e^{j\left(\frac{\pi}{2} m_{v} \cos \theta+2 \theta\right)} d \theta
$$

Thus, the maximum convergence speed with $150 \mathrm{~Hz}$ carrier frequency can be represented by $\Delta V_{c l 150 \mathrm{~Hz} \text { max }}$ as follows:

$$
\begin{aligned}
\Delta V_{c l 150 H z \max }= & \Delta V_{c l x 150 H z \max }-\Delta V_{c l x 150 H z \min } \\
= & \frac{I_{s m}}{\omega C} \frac{\pi}{2} \times A_{1(-2)}\left[\cos \left(\theta_{c x 2}-3 \theta_{v}+\theta\right)\right. \\
& \left.-\cos \left(\theta_{c x 1}-3 \theta_{v}+\theta\right)\right]
\end{aligned}
$$

Normalized by $I_{s m} / \omega C$, the relationship of $\Delta V_{c l 150 \mathrm{Hzmax}}{ }^{*}, m_{v}$ and $\theta$ is illustrated in Fig. 6, where $\theta_{c 1}=0, \theta_{v}=0$, and $N=20$. Fig. 6 shows that, for the traditional CPS-PWM based balancing strategy, the convergence speed is mainly determined by $m_{v}$. Compared to the logic-based CPS-PWM based strategy, when the modulation index $m_{v}$ is higher than 0.75 , its normalized convergence speed is always above 0.3 . Therefore, $\Delta V_{\max }{ }^{*}=0.3$ is taken as the lowest acceptable convergence speed of the proposed balancing strategy.

It can be calculated from (11) or read from Fig. 5(b) that the logicprocessed CPS-PWM scheme based voltage balancing strategy reaches a normalized convergence speed of 0.3 when $\theta \approx \pm \pi / 3$ and $\pm 2 \pi / 3$. Hence the conclusion can be drawn that the application range of power factor angle is $\theta \in[-\pi / 3, \pi / 3]\|[-\pi,-2 \pi / 3]\|[2 \pi / 3, \pi]$. Fig. 7 illustrates the application ranges. Thus, it can be inferred that the proposed strategy is especially suitable for the HVDC

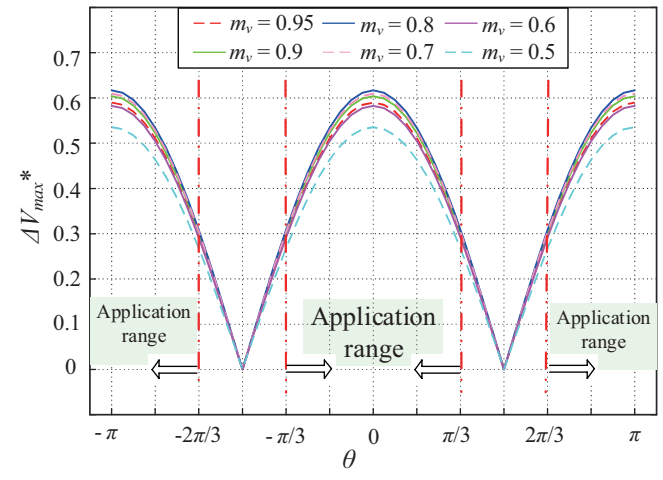

Fig. 7. Application ranges of power factor angle for logic-processed CPS-PWM scheme based balancing strategy.

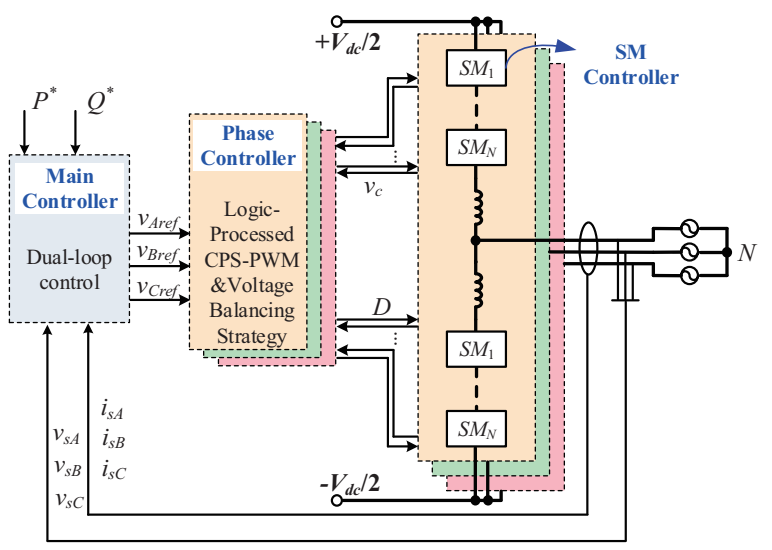

Fig. 8. The three-tier control architecture for logic-processed CPS-PWM based balancing strategy.

transmission, which mainly deals with active power and has a modulation index higher than 0.75 .

\section{Implementation of the Logic-Processed CPS-PWM Based Balancing Strategy}

In the logic-processed CPS-PWM scheme, the manipulation of driving signals should be achieved without affecting the synchronization of switching actions among SMs. Otherwise, distorted voltage levels and defected arm currents will be induced [16]. As demonstrated in Fig. 8, a three-tier control system architecture is proposed. The control system is divided into three parts: the main controller, phase controllers and submodule controllers. The main controller is one DSP controller, which is responsible for the high-level controls, such as active and reactive power control.

The phase controller utilizes FPGA as the control unit, which carries out the logic-processed CPS-PWM scheme based capacitor voltage balancing strategy. Since the generation and manipulation of driving signals are centralized in the middle-tier controller, the synchronization of switching actions is naturally guaranteed. Moreover, the logic process of driving signals is easy and cost-effective in FPGAs. The SM controllers only receive control signals from the phase controllers and report the capacitor voltage to the phase controllers. 
TABLE I

Parameters of Simulation and Prototype

\begin{tabular}{lcc}
\hline \hline Parameters & Simulation & Prototype \\
\hline DC Bus Voltage $\left(V_{d c}\right)$ & $19 \mathrm{kV}$ & $800 \mathrm{~V}$ \\
Apparent Power $(S)$ & $2 \mathrm{MVA}$ & $6 \mathrm{kVA}$ \\
Line to Line Voltage $\left(V_{l-l}, \mathrm{rms}\right)$ & $10 \mathrm{kV}$ & $400 \mathrm{~V}$ \\
Output Frequency $(f)$ & $50 \mathrm{~Hz}$ & $50 \mathrm{~Hz}$ \\
Carrier Frequency $\left(f_{c}\right)$ & $450 \mathrm{~Hz} / 150 \mathrm{~Hz}$ & $450 \mathrm{~Hz}$ \\
Number of SMs in each arm $(N)$ & 20 & 8 \\
Arm Inductance $(L)$ & $40 \mathrm{mH}$ & $40 \mathrm{mH}$ \\
SM Capacitance $(C)$ & $3 \mathrm{mF}$ & $3 \mathrm{mF}$ \\
Rated Capacitor Voltage $\left(V_{c}\right)$ & $950 \mathrm{~V}$ & $100 \mathrm{~V}$ \\
\hline \hline
\end{tabular}

\section{Simulation and Experimental Verification}

In order to verify the theoretical analysis, a 21-level threephase grid-connected inverter is built in MATLAB Simulink and a 9-level three-phase grid connected prototype is established. The parameters of simulation and prototype are shown in Table I. And the carrier frequency (switching frequency) is set as $450 \mathrm{~Hz}$ for both simulation and experiment.

\section{A. Simulation Results}

Dual-loop control is adopted in the simulation, where the outer loop controls the active and reactive power and the inner loop controls the active and reactive current. The carrier phase angle difference for the logic processed driving signals is selected according to [14], so new driving signals with proper charging ability can be synthesized.

Simulation results with different power factor angles are presented in Fig. 9(a)-(c). The output voltage, output current, enable flag and execution flag of balancing strategy, and the lower arm capacitor voltages of phase A are presented successively. In order to verify the effectiveness of the balancing strategy, the capacitor voltages should be diverse at the initial state. Therefore, the balancing strategy is disabled at first, and the logic processed driving signals are intentionally assigned to specific SMs to charge and discharge the capacitors. This process is not illustrated in Fig. 9. After a preset timespan, the SMs are in unbalance state and they are re-assigned with their original driving signals. At the meantime, the balancing strategy is still disabled. As observed in Fig. 9(a)-(c), with the original high frequency driving signals, the capacitor voltages keep unchanged. This is because there is little difference in the charging ability of original driving signals, which verifies the theoretical analysis.

The balancing strategy is enabled at $0.3 \mathrm{~s}$. And it can be seen from Fig. 9(a)-(c) that the capacitor voltages convergence speeds are different with different power factor angles. Fig. 9(d) is the simulation result of the traditional CPS-PWM scheme based balancing strategy with $150 \mathrm{~Hz}$ carrier frequency, and its power factor angle is $\pi / 3$.

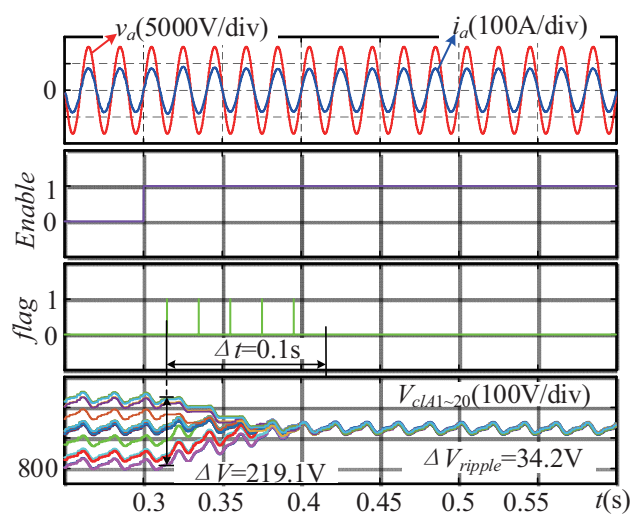

(a)

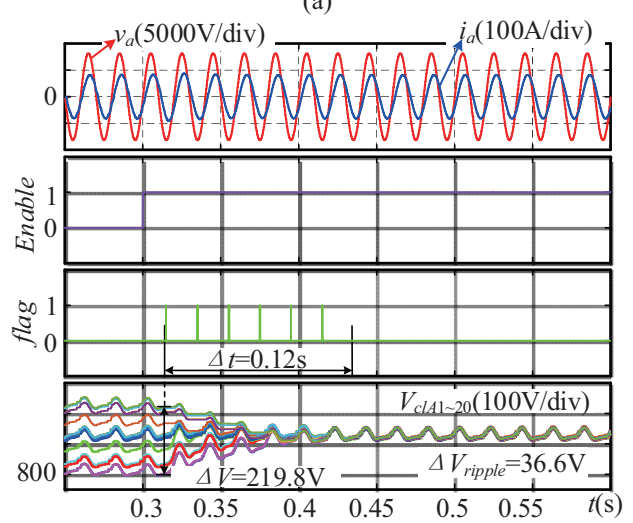

(b)

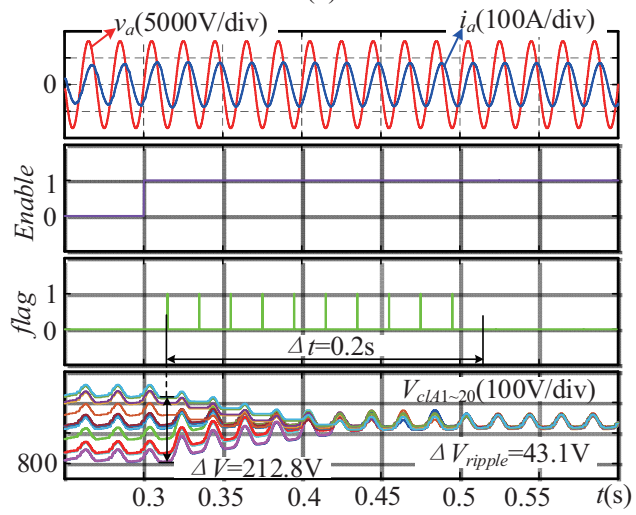

(c)

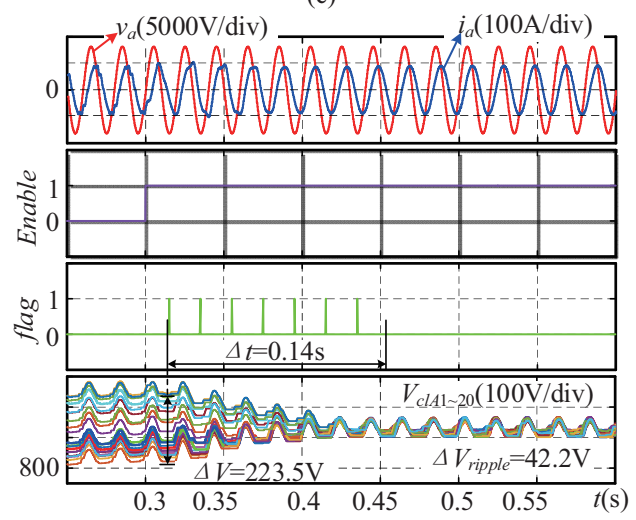

(d)

Fig. 9. Simulation results. (a) power factor angle $\theta=0$ with $450 \mathrm{~Hz}$ logic processed CPS-PWM, (b) $\theta=\pi / 6$ with $450 \mathrm{~Hz}$ logic processed CPS-PWM, (c) $\theta=\pi / 3$ with $450 \mathrm{~Hz}$ logic processed CPS-PWM, (d) $\theta=\pi / 3$ with $150 \mathrm{~Hz}$ traditional CPS-PWM. 
TABLE II

Comparison of Simulation Results

\begin{tabular}{lcccc}
\hline \hline Balancing Strategy & \multicolumn{2}{c}{ Logic Processed CPS-PWM } & $\begin{array}{c}\text { Traditional } \\
\text { CPS-PWM }\end{array}$ \\
\hline Carrier Frequency $\left(f_{c}\right)$ & & $450 \mathrm{~Hz}$ & & $150 \mathrm{~Hz}$ \\
Power Factor Angle $(\theta)$ & 0 & $\pi / 6$ & $\pi / 3$ & $\pi / 3$ \\
Voltage Variation $(\Delta V)$ & $220.3 \mathrm{~V}$ & $219.8 \mathrm{~V}$ & $212.8 \mathrm{~V}$ & $223.5 \mathrm{~V}$ \\
Convergence Time $(\Delta t)$ & $0.1 \mathrm{~s}$ & $0.12 \mathrm{~s}$ & $0.2 \mathrm{~s}$ & $0.14 \mathrm{~s}$ \\
Voltage Ripple $\left(\Delta V_{\text {ripple }}\right)$ & $34.2 \mathrm{~V}$ & $36.6 \mathrm{~V}$ & $43.1 \mathrm{~V}$ & $42.2 \mathrm{~V}$ \\
\hline \hline
\end{tabular}

Key performance characters of the simulation results are concluded in Table II. When the power factor angle $\theta$ changes from 0 to $\pi / 6$, i.e., active power is still the major power component, the convergence speed difference is small. However, when reactive power becomes the major power component (power factor angle $\theta$ changes to $\pi / 3$ ), the convergence time is two times longer than that with $\theta=0$. It indicates that the convergence speed drops quickly with the increase of reactive power. Compared with the traditional CPSPWM scheme based balancing strategy (seven fundamental periods), the convergence speed of logic processed CPS-PWM scheme based balancing strategy is much slower when power factor angle reaches $\pi / 3$ (ten fundamental periods). This result verifies the proposed application ranges of power factor angle in Section III.

\section{B. Experimental Results}

Similarly, the logic-processed voltage balancing strategy is disabled at first, and the driving signal synthesized by logic $O R$ process of the 1st and the 5th carriers is assigned to $\mathrm{SM}_{1}$, and the driving signal synthesized by logic $A N D$ process is assigned to $\mathrm{SM}_{8}$. Besides, the rest SMs' driving signals remain unchanged. Consequently, the capacitor voltages of $\mathrm{SM}_{1}$ and $\mathrm{SM}_{8}$ diverge from their rated value.

Three cases are studied in this section, i.e., the power factor angle equals to $0, \pi / 6$ and $\pi / 3$. The experimental results are presented in Fig. 10(a), (b), (c). The output voltage, output current, capacitor voltages of $\mathrm{SM}_{1}, \mathrm{SM}_{5}$ and $\mathrm{SM}_{8}$ in the lower arm of phase A, execution flag and enable flag of the balancing strategy are depicted in sequence in each figure. It should be noticed that, in order to keep a high convergence speed and avoid the possible voltage oscillations, the selection of carrier phase angle difference for logic processing is divided into three regions according to the voltage divergence degree as introduced in [14]. Therefore, the convergence time is indicated by the execution flag of the outermost region flag 1 and the innermost region flag 3. The key experimental results are concluded in Table III. It is consistent with the analysis and simulation that the convergence time increases with the increase of reactive power component. And when the power factor angle $\theta$ reaches $\pi / 3$, the convergence speed drops a lot compared to the condition when $\theta=0$ (from five fundamental periods to fifteen fundamental periods).

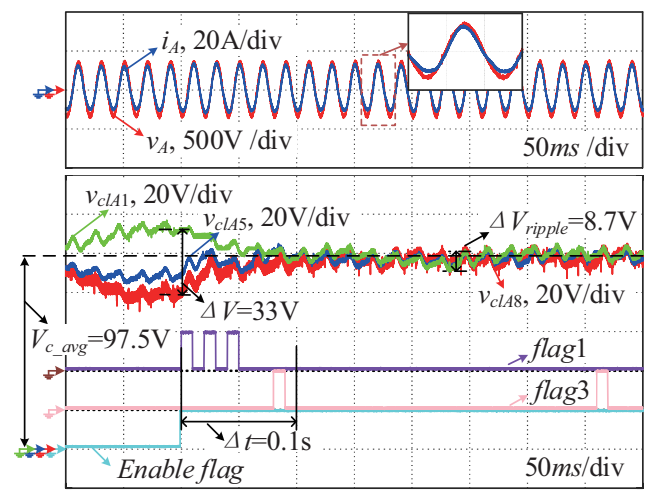

(a)

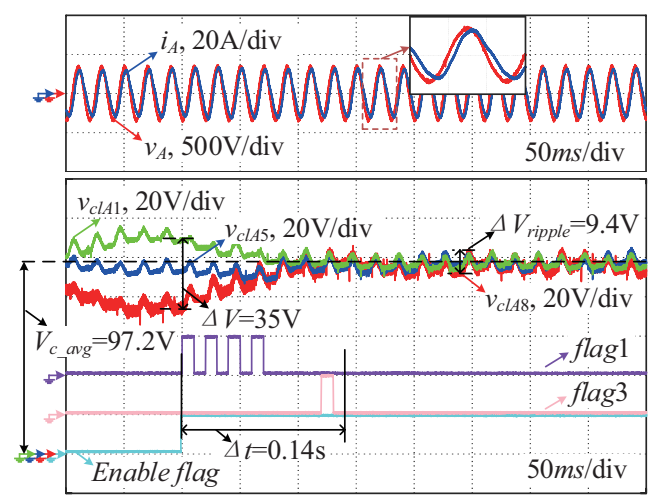

(b)

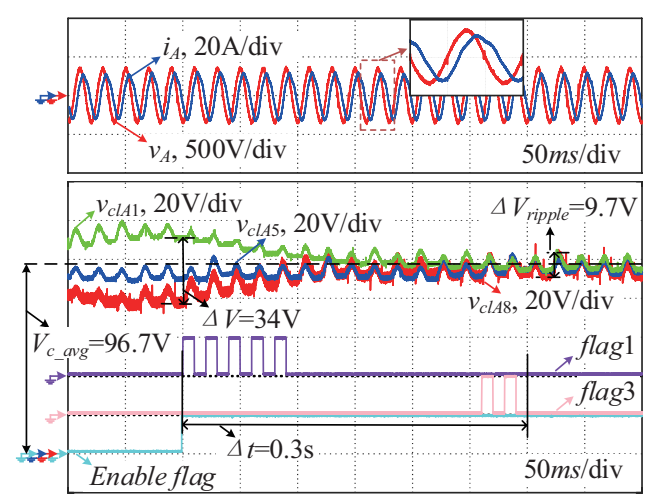

(c)

Fig. 10. Experimental results for $450 \mathrm{~Hz}$ logic-processed CPS-PWM based balancing strategy, (a) power factor angle $\theta=0$, (b) $\theta=\pi / 6$, (c) $\theta=\pi / 3$.

TABLE III

COMPARISON OF EXPERIMENTAL RESUltS

\begin{tabular}{lccc}
\hline \hline Balancing Strategy & \multicolumn{3}{c}{ Logic Processed Scheme With } \\
FFSA \\
\hline Carrier Frequency $\left(f_{c}\right)$ & \multicolumn{3}{c}{$450 \mathrm{~Hz}$} \\
Power Factor Angle $(\theta)$ & 0 & $\pi / 6$ & $\pi / 3$ \\
Voltage Variation $(\Delta V)$ & $33 \mathrm{~V}$ & $35 \mathrm{~V}$ & $34 \mathrm{~V}$ \\
Convergence Time $(\Delta t)$ & $0.1 \mathrm{~s}$ & $0.14 \mathrm{~s}$ & $0.3 \mathrm{~s}$ \\
Voltage Ripple $\left(\Delta V_{\text {ripple }}\right)$ & $8.7 \mathrm{~V}$ & $9.4 \mathrm{~V}$ & $9.5 \mathrm{~V}$ \\
\hline \hline
\end{tabular}




\section{CONCLUSION}

The convergence speed and application ranges of the logicprocessed CPS-PWM scheme based balancing strategy is analyzed in this paper. It is revealed through mathematical analysis that the convergence speed is mainly determined by the power factor of MMC. And by comparing with the normal CPS-PWM scheme based balancing strategy, the application ranges of power factor angle for the proposed balancing strategy is derived, i.e., $[-\pi / 3, \pi / 3]\|[-\pi,-2 \pi / 3]\|[2 \pi / 3, \pi]$. It indicates that this strategy is especially suitable for MMCHVDC transmissions, which mainly deals with active power. Besides, with the proposed three-tier control system, CPSPWM and logic process are centralized in the phase-controllers (FPGA). Therefore, the synchronization of switching actions is guaranteed naturally. The theoretical analysis is validated by both simulation and experimental results.

\section{REFERENCES}

[1] M. A. Perez, S. Bernet, J. Rodriguez, S. Kouro and R. Lizana, "Circuit topologies, modeling, control schemes, and applications of modular multilevel converters," IEEE Trans. Power Electron., vol. 30, no. 1, pp. 4-17, Jan. 2015.

[2] A. Nami, J. Liang, F. Dijkhuizen and G. D. Demetriades, "Modular multilevel converters for HVDC applications: Review on converter cells and functionalities," IEEE Trans. Power Electron., vol. 30, no. 1, pp. 18-36, Jan. 2015.

[3] H. Alyami and Y. Mohamed, "Review and development of MMC employed in VSC-HVDC systems," in Proc. IEEE CCECE, DOI: 10.1109/CCECE.2017.7946676, pp. 1-6, 2017.

[4] M. Hagiwara, R. Maeda and H. Akagi, "Control and analysis of the modular multilevel cascade converter based on double-star choppercells (MMCC-DSCC)," IEEE Trans. Power Electron., vol. 26, no. 6, pp. 1649-1658, Jun. 2011.

[5] R. Lizana, M. A. Perez, D. Arancibia, J. R. Espinoza and J. Rodriguez, "Decoupled current model and control of modular multilevel converters," IEEE Trans. Ind. Electron., vol. 62, no. 9, pp. 5382-5392, Sept. 2015.

[6] R. Zeng, L. Xu, L. Yao and S. J. Finney, "Analysis and control of modular multilevel converters under asymmetric arm impedance conditions," IEEE Trans. Ind. Electron., vol.63, no.1, pp.71-81, Jan. 2016.

[7] M. Hagiwara and H. Akagi, "Control and experiment of pulsewidthmodulated modular multilevel converters," IEEE Trans. Power Electron., vol. 24, no. 7, pp. 1737-1746, Jul. 2009.

[8] S. Fan, K. Zhang, J. Xiong and Y. Xue, "An improved control system for modular multilevel converters with new modulation strategy and voltage balancing control," IEEE Trans. Power Electron., vol. 30, no. 1, pp. 358-371, Jan. 2015.

[9] A. Lesnicar and R. Marquardt, "An innovative modular multilevel converter topology suitable for a wide power range," in Proc. IEEE Power Tech. Conf., vol. 3, pp. 6, Jun. 2003, doi: 10.1109/PTC.2003.1304403.

[10] Q. Tu, Z. Xu and L. Xu, "Reduced switching-frequency modulation and circulating current suppression for modular multilevel converters," IEEE Trans. Power Del., vol. 26, no. 3, pp. 2009-2017, July 2011.

[11] H. Peng, Y. Wang, Z. Lv, Y. Deng, X. He and R. Zhao, "Capacitor voltage balancing based on fundamental frequency sorting algorithm for modular multilevel converter," in Proc. IEEE Energy Convers. Congr. Expo., Sept. 2014, pp. 1639-1644, doi: 10.1109/ECCE.2014.6953614.

[12] H. Peng, R. Xie, K. Wang, Y. Deng, X. He and R. Zhao, "A capacitor voltage balancing method with fundamental sorting frequency for modular multilevel converters under staircase modulation," IEEE Trans. Power Electron. vol. 31, no. 11, pp. 7809-7822, Nov. 2016.

[13] G. Chen, H. Peng, R. Zeng, Y. Hu and K. Ni, "A fundamental frequency sorting algorithm for capacitor voltage balance of modular multilevel converter with low frequency carrier-phase-shift modulation," IEEE $J$. Emerging Sel. Topics Power Electron., vol. 6, no. 3, pp. 1595-1604, Sept. 2018.
[14] K. Wang, Y. Deng, H. Peng, G. Chen, G. Li, and X. He, "An improved CPS-PWM scheme based voltage balancing strategy for MMC with fundamental frequency sorting algorithm," IEEE Trans. Ind. Electron., vol. 66, no. 3, pp. 2387-2397, Mar. 2019.

[15] L. Zhou, K. Wang, Y. Deng, Y. Lu, C. Wang and F. Xu, "Convergence speed analysis of capacitor voltage balancing strategy for $\mathrm{MMC}$ with logic processed CPS-PWM scheme," in Proc. 2018 IEEE Int. Power Electron. Appl. Conf. Expo. (PEAC), Nov. 2018, pp. 1-6, doi: 10.1109/ PEAC.2018.8590429.

[16] S. Huang, R. Teodorescu and L. Mathe, "Analysis of communication based distributed control of MMC for HVDC," in 2013 15th Eur. Conf. Power Electron. Appl. (EPE), Lille, 2013, pp. 1-10.doi: 10.1109/ EPE.2013.6634711.

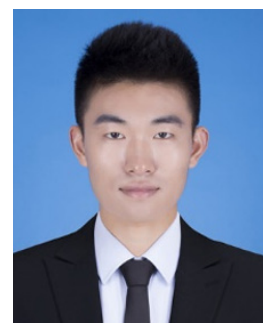

Kun Wang received the B.E.E. degree from the University of Electronic Science and Technology of China, Chengdu, China, in 2013. He is currently working toward the Ph.D. degree from the College of Electrical Engineering, Zhejiang University, Hangzhou, China. His current research interests include modular multilevel converters, dc-dc converters and grid-connected photovoltaic systems.

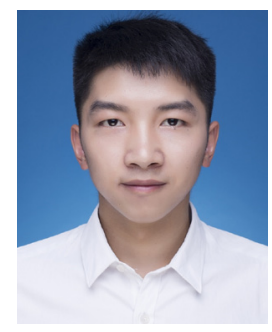

Leyuan Zhou received B.Eng from Nanjing University of Science and Technology, China, in 2015. He is currently working towards his M.S. degree in Power Electronics and Motor Drives. His current research interests include modular multilevel converters (MMC) and the inter-connection of multi-end in the distribution network.

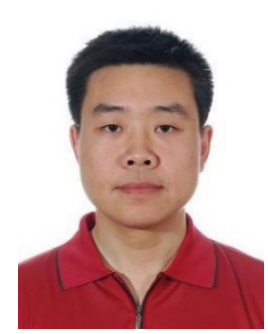

Yan Deng received the B.E.E. degree from the Department of Electrical Engineering, Zhejiang University, Hangzhou, China, in 1994, and the Ph.D. degree in power electronics and electric drives from the College of Electrical Engineering, Zhejiang University, in 2000

Since 2000 , he has been a faculty member at Zhejiang University, teaching and conducting research on power electronics. He is currently a professor with Zhejiang University. His research interests are topologies and control for switch-mode power conversion.

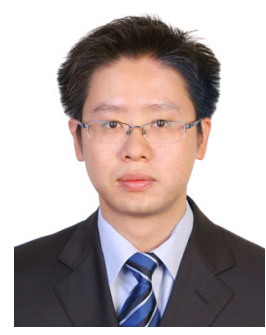

Yi Lu received his B.S. degree in Electrical Engineering from North China Electric Power University, Baoding, China, in 2001. He received his M.S. and Ph.D. degrees in Intelligence Engineering and Electrical Engineering from the University of Liverpool, Liverpool, U.K., in 2002 and 2007, respectively. In 2008, he joined the State Grid Zhejiang Electric Power Research Institute, Hangzhou, China. He is a senior engineer, and his research interests include HVDC and FACTS. 


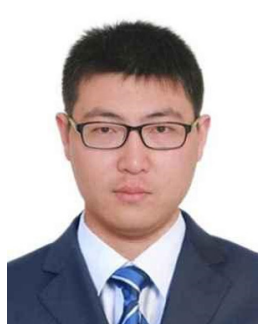

Chaoliang Wang received his B.S. and M.S. degrees in Electrical Engineering from North China Electric Power University, Beijing, China, in 2011 and 2014, respectively. Since 2014, he has been an engineer at State Grid Zhejiang Electric Power Research Institute, Hang Zhou, China. His current research interests include HVDC and FACTS.

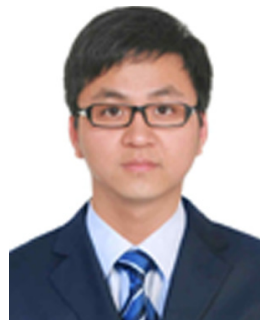

Feng Xu was born in Zhejiang, China, in February 1988. He received the B.S. and Ph.D. degrees in Electrical Engineering from Zhejiang University, Hangzhou, China, in 2010 and 2015 respectively. Currently, he works in State Grid Zhejiang Electric Power Research Institute. His research focus is on high power electronics technology, LCC-HVDC transmission system, VSC-HVDC transmission system, and DC grid. 